DTP-00/11

hep-th/0002065

\title{
First order quantum corrections to the classical reflection factor of the sinh-Gordon model
}

\author{
A. Chenaghlou ${ }^{a}$, 7 and E. Corrigan ${ }^{b}$, \\ a Mathematical Sciences, University of Durham, Durham DH1 3LE, UK \\ ${ }^{b}$ Mathematics, University of York, Heslington, York YO10 5DD, UK
}

\begin{abstract}
The sinh-Gordon model is restricted to a half-line by boundary conditions maintaining integrability. A perturbative calculation of the reflection factor is given to one loop order in the bulk coupling and to first order in the difference of the two parameters introduced at the boundary, providing a further verification of Ghoshal's formula. The calculation is consistent with a conjecture for the general dependence of the reflection factor on the boundary parameters and the bulk coupling.
\end{abstract}

\footnotetext{
${ }^{1}$ alireza. chenaghlou@durham.ac.uk

2 ec9@york.ac.uk
} 


\section{Introduction}

Over the last few years, following the pioneering ideas of Ghoshal and Zamolodchikov, and others [1 5], much work has been done to investigate integrable quantum field theory with a boundary. In particular, the affine Toda field theories have offered a surprisingly rich structure which is just beginning to be understood. The classical affine Toda field theories are known to remain integrable in the presence of certain (generally quite restricted) boundary conditions confining them to a half-line, or to an interval [6-15]. The corresponding quantum field theories are hardly explored although there

has been progress in certain cases associated with the $a_{n}^{(1)}$ class of models [6, 16 [18]. The first of these $(n=1)$ is the sinh-Gordon model and, unlike all the others has a set of integrable boundary conditions depending on a pair of extra parameters, the socalled boundary parameters. However, even in this case, it remains to be seen precisely how the two boundary parameters influence quantities of interest such as the reflection factor.

The sinh-Gordon model is related to the sine-Gordon model and its reflection factors are related to the reflection factors of the lightest breather in the sine-Gordon model. Indeed, the results obtained by Ghoshal and Zamolodchikov [1, 2] allow a determination of the general form of the sinh-Gordon reflection factor and this is a very useful piece of information. However, apart from two special cases (Neumann and Dirichlet boundary conditions) Ghoshal and Zamolodchikov's formulae fail to provide a relationship between the reflection factors and the boundary parameters themselves which appear as the data in a Lagrangian formulation of the model.

Recently, it was noticed [19] that for certain ranges of the boundary parameters in the sinh-Gordon model there are real periodic classical finite-energy solutions called boundary breathers. The sinh-Gordon model has no real finite-energy solutions at all on the whole line (other than $\phi=0$ ) but, once there is a boundary singularities may be hidden behind it allowing solutions restricted to a half-line to have finite energy. The existence of periodic solutions allows a semi-classical quantization determining the spectrum of boundary bound states. Once the spectrum of bound state energies is known it may be compared with a boundary bootstrap calculation of the energies of the same states and hence may be used, in principle, to find a relationship between the Lagrangian boundary parameters and the data in Ghoshal's formula. In fact this is complicated and was carried out in 19 for the simpler situation in which the two boundary parameters are equal and the $\phi \rightarrow-\phi$ symmetry of the bulk theory is preserved. The corresponding calculation in the more general case is not yet completed. The semi-classical calculation may turn out to be exact (as it did for the energy spectrum of the breathers in the bulk sine-Gordon model itself; for a review see [20]) but, failing a proof of exactness the results may be checked against low-order perturbation theory [21 24].

If the two boundary parameters are different and the $\phi \rightarrow-\phi$ symmetry is broken, the perturbation theory becomes substantially more complicated: the lowest energy static background is no longer the configuration $\phi=0$ and therefore the perturbation theory must be developed within a non-trivial background, leading to additional cubic 
and higher odd order couplings as well as a substantially more intricate propagator. Nevertheless, as will be shown below, provided calculations are restricted to first order in the difference of the two boundary parameters, some of the complications disappear and we are able to calculate the correction to the reflection factor at one loop. The result we obtain is consistent with a conjecture for the relationship between the boundary parameters and Ghoshal's formula in the more general setting. A full discussion, to all orders in the difference of the boundary parameters, even at one loop order in the bulk coupling, must be postponed.

\section{2 sinh-Gordon model}

The sinh-Gordon theory corresponds to the affine Toda field theory associated with $a_{1}^{(1)}$. The bulk Lagrangian density of the theory is:

$$
\mathcal{L}=\frac{1}{2} \partial_{\mu} \phi \partial^{\mu} \phi-V(\phi)
$$

where

$$
V(\phi)=\frac{2 m^{2}}{\beta^{2}} \cosh (\beta \alpha \phi)
$$

The real constants $m$ and $\beta$ provide a mass scale and a coupling constant, respectively, and it is customary in affine Toda field theory to choose $\alpha=\sqrt{2}$.

Assuming two sinh-Gordon particles of relative rapidity $\Theta$ scatter from each other elastically, the bulk S-matrix characterizing this process is given by 25 27]

$$
S(\Theta)=-\frac{1}{(B)(2-B)}
$$

where

$$
B=\frac{1}{2 \pi} \frac{\beta^{2}}{1+\beta^{2} / 4 \pi}
$$

and the symbol ( ) denotes the hyperbolic building block:

$$
(x)=\frac{\sinh \left(\Theta / 2+\frac{i \pi x}{4}\right)}{\sinh \left(\Theta / 2-\frac{i \pi x}{4}\right)}
$$

The S-matrix is invariant under the following weak-strong coupling transformation

$$
\beta \rightarrow 4 \pi / \beta
$$

a property known as weak-strong coupling duality.

A sinh-Gordon theory on the half-line is described by the following Lagrangian density:

$$
\overline{\mathcal{L}}=\theta(-x) \mathcal{L}-\delta(x) \mathcal{B}
$$


where $\mathcal{B}$ is regarded as a functional of the field only, not its time derivative. Moreover, the generic form of $\mathcal{B}$ is given by [1]

$$
\mathcal{B}=\frac{m}{\beta^{2}}\left(\sigma_{0} e^{-\frac{\beta}{\sqrt{2}} \phi}+\sigma_{1} e^{\frac{\beta}{\sqrt{2}} \phi}\right)
$$

Note, the coefficients $\sigma_{0}$ and $\sigma_{1}$ are a pair of real numbers, essentially free (but see [7, 10]), which represent the extra parameters permitted at the boundary $x=0$.

The equation of motion and the boundary condition for the sinh-Gordon model on the half-line become (after rescaling the mass scale to unity):

$$
\begin{array}{ll}
\partial^{2} \phi=-\frac{\sqrt{2}}{\beta}\left(e^{\sqrt{2} \beta \phi}-e^{-\sqrt{2} \beta \phi}\right) & x<0 \\
\frac{\partial \phi}{\partial x}=-\frac{\sqrt{2}}{\beta}\left(\sigma_{1} e^{\beta \phi / \sqrt{2}}-\sigma_{0} e^{-\beta \phi / \sqrt{2}}\right) & x=0
\end{array}
$$

\section{Reflection factor}

When a sinh-Gordon particle is moving towards the boundary located at $x=0$ it will reflect elastically from it meaning that the in- and out- one particle states, conveniently labelled by rapidity, will be related by a reflection factor

$$
\left|\theta>_{\text {out }}=K(\theta)\right|-\theta>_{\text {in }} .
$$

The general form of this reflection factor is known although its detailed dependence on the boundary parameters $\sigma_{0}$ and $\sigma_{1}$ appearing in (2.8) is known only in special cases. The sequence of arguments determining its form is somewhat indirect, stemming from the work of Ghoshal and Zamolodchikov [1] as follows. Solving the boundary YangBaxter equation, and using general constraints implementing unitarity and a form of crossing symmetry, it proved possible to calculate the reflection factor for the sineGordon soliton. Since breathers are soliton-anti-soliton bound states, a subsequent set of calculations using the boundary bootstrap led Ghoshal [2] to conjecture reflection factors for each member of the full tower of breathers. Finally, the reflection factor for the lightest breather is supposed to be identical with that of the sinh-Gordon particle provided the sine-Gordon coupling $\beta$ is replaced by $i \beta$.

Thus, suitably transformed in the manner described, Ghoshal's formula for the sinh-Gordon reflection factor is given by:

$$
K_{q}(\theta)=\frac{(1)(2-B / 2)(1+B / 2)}{\left(1-E\left(\sigma_{0}, \sigma_{1}, \beta\right)\right)\left(1+E\left(\sigma_{0}, \sigma_{1}, \beta\right)\right)\left(1-F\left(\sigma_{0}, \sigma_{1}, \beta\right)\right)\left(1+F\left(\sigma_{0}, \sigma_{1}, \beta\right)\right)}
$$

Actually, Ghoshal's notation was a little different and made use of two other quantities $\eta, \vartheta$ defined by $E=B \eta / \pi, F=i B \vartheta / \pi$.

There are special cases in which $E$ and $F$ have been conjectured. For example, the Neumann boundary condition which is defined by

$$
\frac{\partial \phi}{\partial x}=0 \quad \text { when } \quad x=0
$$


has been argued by Ghoshal and Zamolodchikov to need a reflection factor containing

$$
F=0, \quad E=1-B / 2 .
$$

More recently [19, the boundary breather states of the sinh-Gordon model restricted to a half-line were investigated and their energy spectrum calculated in two ways. First, by using the bootstrap equations, and then by finding a set of periodic finite-energy solutions which could be quantized using a WKB approximation. Insisting that the two methods agreed with each other provided strong evidence for a relationship between the boundary parameters, the bulk coupling constant, and the parameters appearing in the reflection factor. For technical reasons this work was restricted to the case $\sigma_{0}=\sigma_{1}$ but, nevertheless, yielded an expression for $E$, with $F=0$. Specifically, setting $\sigma_{0}=\sigma_{1}=\cos a \pi$ and restricting $a$ to the range $1>a>1 / 2, E$ is given by,

$$
E=2 a(1-B / 2)
$$

In the limit $a \rightarrow 1 / 2$ from above, this is in agreement with (3.4).

Both of these conjectures are underpinned by low order perturbation theory. Kim 21] has calculated the one loop correction to the classical Neumann boundary condition reflection factor and found agreement with Ghoshal's formula to $O\left(\beta^{2}\right)$. On the other hand, a more general calculation was carried out, also to one loop, agreeing with the conjecture (3.5), and indeed, preceding it 23]. In fact, the perturbative calculation in 23] agrees with (3.5) for $a$ in the range $1>a>0$.

However, when $\sigma_{0} \neq \sigma_{1}$ the situation becomes much more complicated. Indeed, all that is known up to now is the behaviour of $E$ and $F$ in the limit $\beta \rightarrow 0$, deduced from a direct calculation of the classical reflection factor in the general case [7]. To describe this limit it is convenient to set $\sigma_{0}=\cos a_{0} \pi$ and $\sigma_{1}=\cos a_{1} \pi$, with $\left|a_{i}\right| \leq 1, i=0,1$. Then,

$$
E\left(0, \sigma_{0}, \sigma_{1}\right)=a_{0}+a_{1}, \quad F\left(0, \sigma_{0}, \sigma_{1}\right)=a_{0}-a_{1} .
$$

and, the classical reflection factor itself is given in term of the basic factors (2.5) by

$$
K_{c}(\theta)=-\frac{(1)^{2}}{\left(1-a_{0}-a_{1}\right)\left(1+a_{0}+a_{1}\right)\left(1-a_{0}+a_{1}\right)\left(1+a_{0}-a_{1}\right)} .
$$

The expression (3.7) is an essential ingredient of the basic two-point function, or propagator, which takes the form [23],

$$
\begin{aligned}
G\left(x, t ; x^{\prime}, t^{\prime}\right)=\iint \frac{d \omega}{2 \pi} & \frac{d k}{2 \pi} \frac{i e^{-i \omega\left(t-t^{\prime}\right)}}{\left(\omega^{2}-k^{2}-4+i \rho\right)} f\left(-k, x^{\prime}\right) \\
& \times\left(f(k, x) e^{i k\left(x-x^{\prime}\right)}+K_{c} f(-k, x) e^{-i k\left(x+x^{\prime}\right)}\right)
\end{aligned}
$$

where

$$
f(k, x)=\frac{i k-2 \operatorname{coth} 2\left(x-x_{0}\right)}{i k+2} .
$$

The parameter $x_{0}$ enters the static background

$$
e^{\beta \phi_{0} / \sqrt{2}}=\frac{1+e^{2\left(x-x_{0}\right)}}{1-e^{2\left(x-x_{0}\right)}}
$$


and determines the point at which the background becomes singular. For this reason, it is crucial that $x_{0} \geq 0$. Actually, $x_{0}$ is determined by the boundary condition (2.10) and satisfies

$$
\operatorname{coth} x_{0}=\sqrt{\frac{1+\sigma_{0}}{1+\sigma_{1}}} .
$$

On the understanding that $\sigma_{1} \geq \sigma_{0}$, it is enough to consider this situation since (2.10) is symmetric under $\phi \rightarrow-\phi$ and the interchange of $\sigma_{0}$ with $\sigma_{1}$.

In the expression (3.8) for the propagator, the classical reflection factor appears as the coefficient of the reflection part of the free field two-point function calculated within the classical static background. To check Ghoshal's formula, the strategy introduced by Kim [21] and developed in [23] is to calculate perturbative corrections to the two-point function and then to identify corrections to the classical reflection factor by picking out the coefficient of $e^{-i k\left(x+x^{\prime}\right)}$ as $x, x^{\prime} \rightarrow-\infty$.

\section{First order quantum corrections to the reflection factor}

Previously, as noted above, the first order perturbation calculation was achieved for the specially symmetric case $\sigma_{0}=\sigma_{1}$. In this section, this restriction will be relaxed and enough of the ingredients of the perturbation expansion will be calculated to allow a calculation (in principle) up to $\beta^{2}$. We will discover that generally the theory needs three- and four- point couplings which depend upon the $x$-dependent static background.

Expanding the bulk potential (2.2) around the background solution to the equation of motion, $\phi_{0}(x)$, we derive the three- and four-point couplings:

$$
C_{\mathrm{bulk}}^{(3)}=\frac{2 \sqrt{2}}{3} \beta \sinh \left(\sqrt{2} \beta \phi_{0}\right)
$$

and

$$
C_{\text {bulk }}^{(4)}=\frac{1}{3} \beta^{2} \cosh \left(\sqrt{2} \beta \phi_{0}\right) .
$$

On the other hand, the static background is (3.10). So, after some manipulation, we have

$$
C_{\mathrm{bulk}}^{(3)}=\frac{4 \sqrt{2}}{3} \beta \cosh 2\left(x-x_{0}\right)\left(\operatorname{coth}^{2} 2\left(x-x_{0}\right)-1\right) \text {, }
$$

and

$$
C_{\text {bulk }}^{(4)}=\frac{1}{3} \beta^{2}\left(2 \operatorname{coth}^{2} 2\left(x-x_{0}\right)-1\right) .
$$

In the same manner, using (2.8) we can derive the three- and four-point couplings associated with the boundary term:

$$
C_{\text {boundary }}^{(3)}=\frac{\sqrt{2} \beta}{12}\left(\sigma_{1} e^{\beta \phi_{0} / \sqrt{2}}-\sigma_{0} e^{-\beta \phi_{0} / \sqrt{2}}\right)
$$

and

$$
C_{\text {boundary }}^{(4)}=\frac{\beta^{2}}{48}\left(\sigma_{1} e^{\beta \phi_{0} / \sqrt{2}}+\sigma_{0} e^{-\beta \phi_{0} / \sqrt{2}}\right)
$$


For future reference, it will be useful to know these to first order in the difference of the two boundary parameters $\epsilon=\sigma_{0}-\sigma_{1}$ :

$$
\begin{gathered}
C_{\mathrm{bulk}}^{(3)}=\frac{2 \sqrt{2}}{3} \beta \frac{\epsilon}{1+\sigma_{1}} e^{2 x}+\ldots \\
C_{\mathrm{bulk}}^{(4)}=\frac{1}{3} \beta^{2}+\ldots
\end{gathered}
$$

and similarly,

$$
\begin{gathered}
C_{\text {boundary }}^{(3)}=\frac{\sqrt{2} \beta}{12}\left(-\frac{\epsilon}{1+\sigma_{1}}\right)+\ldots \\
C_{\text {boundary }}^{(4)}=\frac{\beta^{2}}{48}\left(2 \sigma_{1}+\epsilon\right)+\ldots
\end{gathered}
$$

We shall also need the expressions for $f(k, x)(3.9)$ and the classical reflection factor $K_{c}$ (3.7) to the same order in $\epsilon$ since both contribute to the propagator (3.8). In fact,

$$
f(k, x)=1+O\left(\epsilon^{2}\right)
$$

and the classical reflection factor (3.7) reduces to

$$
K_{c}=\frac{i k+2 \sigma}{i k-2 \sigma}+\frac{2 i k}{(i k-2 \sigma)^{2}} \epsilon+O\left(\epsilon^{2}\right) \text {. }
$$

It is also convenient to write

$$
K_{c}=K_{0}+\epsilon K_{1}
$$

where $K_{0}$ is the classical reflection factor when the two boundary parameters are equal.

To calculate quantum corrections to the classical reflection factor at one loop order we use perturbative methods generalised to the affine Toda field theory on a half-line 21 24. (For earlier references on boundary perturbation theory in general see; 28 30 for affine Toda perturbation theory see [31], or the review [32].) The $O\left(\beta^{2}\right)$ correction to $K_{0}$ has been calculated before and the purpose of this article is to calculate the corrections to $K_{1}$ to the same order.

The possible diagrams to $O\left(\beta^{2}\right)$ are:

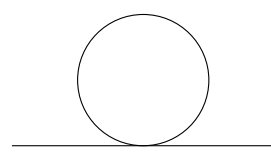

I

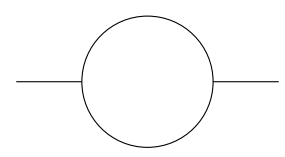

II

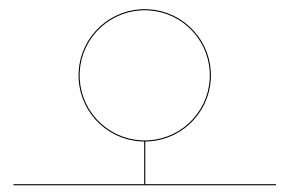

III

Figure 1: Three basic Feynman diagrams in one loop order. 
These will be computed in configuration space noting that each vertex may either be situated at the boundary or within the bulk. In effect, there are ten contributions which need to be calculated.

However, there is a simplifying feature provided we are content to work to first order in $\epsilon$. To recognise this it is enough to note that because the three-point coupling is already $O(\epsilon)$, implying the type II and III diagrams involve $\epsilon^{2}$, only diagrams of type I need concern us.

Thus, there are two contributions which need to be calculated in order to be able to deduce the quantum corrections to the classical reflection factor, both of type I. The first is directly related to the boundary, when the interaction vertex coincides with the boundary, and it takes the form

$$
-\frac{i \beta^{2}}{4}\left(2 \sigma_{1}+\epsilon\right) \int_{-\infty}^{+\infty} d t^{\prime \prime} G\left(x, t ; 0, t^{\prime \prime}\right) G\left(0, t^{\prime \prime} ; 0, t^{\prime \prime}\right), G\left(0, t^{\prime \prime} ; x^{\prime}, t^{\prime}\right),
$$

including the correct coupling constant and combinatorial factors.

The second contribution refers to the bulk potential which means the interaction vertex is located inside the bulk region $x<0$. This contribution is given by

$$
-4 i \beta^{2} \int_{-\infty}^{+\infty} d t^{\prime \prime} \int_{-\infty}^{0} d x^{\prime \prime} G\left(x, t ; x^{\prime \prime}, t^{\prime \prime}\right) G\left(x^{\prime \prime}, t^{\prime \prime} ; x^{\prime \prime}, t^{\prime \prime}\right) G\left(x^{\prime \prime}, t^{\prime \prime} ; x^{\prime}, t^{\prime}\right) .
$$

Again, the combinatorial factor has been included.

Let us first calculate the boundary contribution (4.14). The loop propagator is given to $O(\epsilon)$ by

$$
G\left(0, t^{\prime \prime} ; 0, t^{\prime \prime}\right)=i \iint \frac{d \omega^{\prime \prime}}{2 \pi} \frac{d k^{\prime \prime}}{2 \pi} P_{0}\left(\omega^{\prime \prime}, k^{\prime \prime}\right)\left(1+\frac{i k^{\prime \prime}+2 \sigma_{1}}{i k^{\prime \prime}-2 \sigma_{1}}+\frac{2 i k^{\prime \prime} \epsilon}{\left(i k^{\prime \prime}-2 \sigma_{1}\right)^{2}}\right),
$$

where we have defined

$$
P_{0}\left(\omega^{\prime \prime}, k^{\prime \prime}\right)=\frac{1}{\omega^{\prime \prime 2}-k^{\prime 2}-4+i \rho} .
$$

Note, the integral is clearly divergent but the divergence is removed by a renormalization of the boundary parameter. In effect, rearranging the part of the integrand containing the offending terms as follows,

$$
1+\frac{i k^{\prime \prime}+2 \sigma_{1}}{i k^{\prime \prime}-2 \sigma_{1}}=2+\frac{4 \sigma}{i k^{\prime \prime}-2 \sigma_{1}}
$$

and making a minimal subtraction of the divergent portion, (i.e. deleting the '2'), renders the integral finite.

The part of the integral independent of $\epsilon$ has been calculated before [23], therefore we may write,

$$
G\left(0, t^{\prime \prime} ; 0, t^{\prime \prime}\right)=-\frac{a_{1} \cos a_{1} \pi}{\sin a_{1} \pi}+i \iint \frac{d \omega^{\prime \prime}}{2 \pi} \frac{d k^{\prime \prime}}{2 \pi} P_{0}\left(\omega^{\prime \prime}, k^{\prime \prime}\right) \frac{2 i k^{\prime \prime} \epsilon}{\left(i k^{\prime \prime}-2 \sigma_{1}\right)^{2}},
$$


and concentrate on the $O(\epsilon)$ part.

As far as the remaining integral is concerned, focusing on the energy variable and closing the integration contour in the upper half-plane, we encounter a simple pole at $\sqrt{k^{\prime 2}+4}$. Therefore, we need to evaluate

$$
\frac{1}{2} \int \frac{d k^{\prime \prime}}{2 \pi} \frac{1}{\sqrt{k^{\prime \prime 2}+4}} \frac{2 i k^{\prime \prime}}{\left(i k^{\prime \prime}-2 \sigma_{1}\right)^{2}} \epsilon
$$

The $k^{\prime \prime}$ integration may be performed by closing the contour in the upper (lower) halfplane depending on whether $\sigma_{1}>0\left(\sigma_{1}<0\right)$. However, because of the branch points at $\pm 2 i$ it is convenient to locate the associated branch cuts along the imaginary axis from $\pm 2 i$ to $\infty$. Evaluating the integral and assembling the pieces, we obtain

$$
i \iint \frac{d \omega^{\prime \prime}}{2 \pi} \frac{d k^{\prime \prime}}{2 \pi} P_{0}\left(\omega^{\prime \prime}, k^{\prime \prime}\right) \frac{2 i k^{\prime \prime}}{\left(i k^{\prime \prime}-2 \sigma_{1}\right)^{2}} \epsilon=-\frac{\epsilon}{2} \frac{a_{1}}{\sin ^{3} a_{1} \pi}+\frac{\epsilon \cos a_{1} \pi}{2 \pi} \frac{1}{\sin ^{2} a_{1} \pi} \text {. }
$$

At this stage, the contribution to (4.14) is

$$
\begin{aligned}
& -i \frac{\beta^{2}}{4}\left(2 \sigma_{1}+\epsilon\right)\left(-\frac{a_{1} \cos a_{1} \pi}{\sin a_{1} \pi}-\frac{\epsilon}{2} \frac{a_{1}}{\sin ^{3} a_{1} \pi}+\frac{\epsilon \cos a_{1} \pi}{2 \pi} \frac{1}{\sin ^{2} a_{1} \pi}\right) \\
\times & i \int d t^{\prime \prime} \iint \frac{d \omega}{2 \pi} \frac{d k}{2 \pi} e^{-i \omega\left(t-t^{\prime \prime}\right)} P_{0}(\omega, k) e^{-i k x}\left(\frac{2 i k}{i k-2 \sigma_{1}}+\frac{2 i k \epsilon}{\left(i k-2 \sigma_{1}\right)^{2}}\right) \\
\times & i \iint \frac{d \omega^{\prime}}{2 \pi} \frac{d k^{\prime}}{2 \pi} e^{-i \omega^{\prime}\left(t^{\prime \prime}-t^{\prime}\right)} P_{0}\left(\omega^{\prime}, k^{\prime}\right) e^{-i k^{\prime} x^{\prime}}\left(\frac{2 i k^{\prime}}{i k^{\prime}-2 \sigma_{1}}+\frac{2 i k^{\prime} \epsilon}{\left(i k^{\prime}-2 \sigma_{1}\right)^{2}}\right)
\end{aligned}
$$

The integration over $t^{\prime \prime}$ ensures energy conservation at the interaction vertex and creates a Dirac delta function which immediately removes one of the energy variables, for example $\omega^{\prime}$. The remaining integral over the momenta $k$ and $k^{\prime}$ can be performed by completing the contours in the upper half-plane and taking into account the poles at $k=k^{\prime}=\sqrt{\omega^{2}-4} \equiv \hat{k}$. However, if $\sigma_{1}>0$ it is evident that the expressions for $K_{0}$ and $K_{1}$ have no poles inside the contour. If $\sigma_{1}<0$, there is an additional pole but its contribution turns out to be exponentially decreasing in the asymptotic region $x, x^{\prime} \rightarrow-\infty$.

Finally, we obtain the boundary contribution (4.14) in the form

$$
\begin{array}{r}
-i \frac{\beta^{2}}{4} \int \frac{d \omega}{2 \pi} e^{-i \omega\left(t-t^{\prime}\right)} e^{-i \hat{k}\left(x+x^{\prime}\right)}\left\{\frac{2 a_{1} \cos ^{2} a_{1} \pi}{\sin a_{1} \pi} \frac{1}{\left(i \hat{k}-2 \sigma_{1}\right)^{2}}\right. \\
+\left(\frac{a_{1} \cos a_{1} \pi}{\sin a_{1} \pi}+\frac{a_{1} \cos a_{1} \pi}{\sin ^{3} a_{1} \pi}-\frac{\cos ^{2} a_{1} \pi}{\pi \sin ^{2} a_{1} \pi}\right) \frac{\epsilon}{\left(i \hat{k}-2 \sigma_{1}\right)^{2}} \\
\left.+\frac{4 a_{1} \cos ^{2} a_{1} \pi}{\sin a_{1} \pi} \frac{\epsilon}{\left(i \hat{k}-2 \sigma_{1}\right)^{3}}\right\},
\end{array}
$$

where $\hat{k}=2 \sinh \theta$.

Next, we need to calculate the contribution (4.15) which to $O(\epsilon)$ is: 


$$
\begin{aligned}
& -4 i \beta^{2} \int d t^{\prime \prime} \int_{-\infty}^{0} d x^{\prime \prime} \iint \frac{d \omega}{2 \pi} \frac{d k}{2 \pi} e^{-i \omega\left(t-t^{\prime \prime}\right)} i P_{0}(\omega, k) \\
& \left(e^{-i k\left(x-x^{\prime \prime}\right)}+\frac{i k+2 \sigma_{1}}{i k-2 \sigma_{1}} e^{-i k\left(x+x^{\prime \prime}\right)}+\frac{2 i k \epsilon}{\left(i k-2 \sigma_{1}\right)^{2}} e^{-i k\left(x+x^{\prime \prime}\right)}\right) \\
& \iint \frac{d \omega^{\prime \prime}}{2 \pi} \frac{d k^{\prime \prime}}{2 \pi} i P_{0}\left(\omega^{\prime \prime}, k^{\prime \prime}\right)\left(1+\frac{i k^{\prime \prime}+2 \sigma_{1}}{i k^{\prime \prime}-2 \sigma_{1}} e^{-2 i k^{\prime \prime} x^{\prime \prime}}+\frac{2 i k^{\prime \prime} \epsilon}{\left(i k^{\prime \prime}-2 \sigma_{1}\right)^{2}} e^{-2 i k^{\prime \prime} x^{\prime \prime}}\right) \\
& \iint \frac{d \omega^{\prime}}{2 \pi} \frac{d k^{\prime}}{2 \pi} i P_{0}\left(\omega^{\prime}, k^{\prime}\right) e^{-i \omega^{\prime}\left(t^{\prime \prime}-t^{\prime}\right)} \\
& \left(e^{i k^{\prime}\left(x^{\prime \prime}-x^{\prime}\right)}+\frac{i k^{\prime}+2 \sigma_{1}}{i k^{\prime}-2 \sigma_{1}} e^{-i k^{\prime}\left(x^{\prime \prime}+x^{\prime}\right)}+\frac{2 i k^{\prime} \epsilon}{\left(i k^{\prime}-2 \sigma_{1}\right)^{2}} e^{-i k^{\prime}\left(x^{\prime \prime}+x^{\prime}\right)}\right) .(
\end{aligned}
$$

The integral over $t^{\prime \prime}$ yields a delta function which replaces $\omega^{\prime}$ by $\omega$. Furthermore, to calculate the integration over $x^{\prime \prime}$, it is convenient to use the following device

$$
\int_{-\infty}^{0} d x^{\prime \prime} e^{i k x^{\prime \prime}+\tau x^{\prime \prime}}=\frac{-i}{k-i \tau}
$$

where the small positive quantity $\tau$ will be taken to zero at the final stage of the calculations.

The loop integral which corresponds to the middle propagator of (4.15), is obviously logarithmically divergent and this divergence will be removed by the infinite renormalization of the mass parameter in the bulk potential. So, after making the subtraction and integrating $x^{\prime \prime}$ and $\omega^{\prime \prime}$, and as before concentrating on the $O(\epsilon)$ piece, we obtain the contribution

$$
\begin{aligned}
& -\frac{i}{2} \iiint \frac{d \omega}{2 \pi} \frac{d k}{2 \pi} \frac{d k^{\prime}}{2 \pi} e^{-\imath \omega\left(t-t^{\prime}\right)} e^{-i\left(k x+k^{\prime} x^{\prime}\right)} i P_{0}(\omega, k) i P_{0}\left(\omega, k^{\prime}\right) \\
& \int \frac{d k^{\prime \prime}}{2 \pi} \frac{1}{\sqrt{k^{\prime \prime 2}+4}}\left\{\frac { 2 i k \epsilon } { ( i k - 2 \sigma _ { 1 } ) ^ { 2 } } \frac { i k ^ { \prime \prime } + 2 \sigma _ { 1 } } { i k ^ { \prime \prime } - 2 \sigma _ { 1 } } \left(\frac{1}{-k+k^{\prime}-2 k^{\prime \prime}-i \tau}\right.\right. \\
& +\frac{2 i k^{\prime} \epsilon}{\left(i k^{\prime}-2 \sigma_{1}\right)^{2}} \frac{i k^{\prime \prime}+2 \sigma_{1}}{i k^{\prime \prime}-2 \sigma_{1}}\left(\frac{1}{-k^{\prime}+k-2 k^{\prime \prime}-i \tau}-\frac{1 k^{\prime \prime}+i \tau}{i k^{\prime}-2 \sigma_{1}}\right) \\
& +\frac{2 i k^{\prime \prime} \epsilon}{\left(i k^{\prime \prime}-2 \sigma_{1}\right)^{2}}\left(\frac{1}{k+k^{\prime}-2 k^{\prime \prime}-i \tau}+\frac{1}{k-k^{\prime}-2 k^{\prime \prime}-i \tau} \frac{i k+2 \sigma_{1}}{i k-2 \sigma_{1}}\right) \\
& -\frac{1}{i k^{\prime}-2 \sigma_{1}} \\
& \left.\left.\frac{i k+2 \sigma_{1}}{k-k^{\prime}+2 k^{\prime \prime}+i \tau} \frac{1}{i k-2 \sigma_{1}}-\frac{i k+2 \sigma_{1}}{k+k^{\prime}+2 k^{\prime \prime}+i \tau} \frac{i k^{\prime}+2 \sigma_{1}}{i k-2 \sigma_{1}}\right)\right\} .(4.26)
\end{aligned}
$$

In order to evaluate the integral over $k^{\prime \prime}$, we encounter the following two types:

$$
\int \frac{d k^{\prime \prime}}{2 \pi} \frac{1}{\sqrt{k^{\prime \prime 2}+4}}\left(\frac{i k^{\prime \prime}+2 \sigma_{1}}{i k^{\prime \prime}-2 \sigma_{1}}\right) \frac{1}{\left(k+k^{\prime}-2 k^{\prime \prime}-i \tau\right)}
$$


and

$$
\int \frac{d k^{\prime \prime}}{2 \pi} \frac{1}{\sqrt{k^{\prime \prime 2}+4}} \frac{2 i k^{\prime \prime} \epsilon}{\left(i k^{\prime \prime}-2 \sigma_{1}\right)^{2}} \frac{1}{\left(k+k^{\prime}-2 k^{\prime \prime}-i \tau\right)} .
$$

Both of these may be performed by closing an appropriate contour in the upper halfplane ensuring that it runs around the branch cut located from $k^{\prime \prime}=2 i$ to infinity along the imaginary axis. Note, if $\sigma_{1}>0$, then there is no pole inside the contour; however, if $\sigma_{1}>0$, there is an extra pole but its residue integrated over $k$ and $k^{\prime}$ will give a contribution vanishing in the limit $x, x^{\prime} \rightarrow-\infty$.

For example, the integral (4.28) evaluates to

$$
\begin{aligned}
\int \frac{d k^{\prime \prime}}{2 \pi} & \frac{1}{\sqrt{k^{\prime \prime 2}+4}} \frac{2 i k^{\prime \prime} \epsilon}{\left(i k^{\prime \prime}-2 \sigma_{1}\right)^{2}} \frac{1}{\left(k+k^{\prime}-2 k^{\prime \prime}-i \tau\right)}=\frac{\epsilon}{\pi} \frac{k+k^{\prime}}{\left(i k+i k^{\prime}-4 \sigma_{1}\right)^{2}} \frac{a_{1} \pi}{\sin a_{1} \pi} \\
& -\frac{\epsilon}{\pi} \frac{i \sigma_{1}}{\left(4 \sigma_{1}-i k-i k^{\prime}\right)} \frac{1}{\sin ^{2} a_{1} \pi}+\frac{\epsilon}{\pi} \frac{i \sigma_{1}^{2}}{\left(4 \sigma_{1}-i k-i k^{\prime}\right)} \frac{a_{1} \pi}{\sin ^{3} a_{1} \pi} \\
+ & \frac{\epsilon}{\pi} \frac{2 i\left(k+k^{\prime}\right)}{\left(i k+i k^{\prime}-4 \sigma_{1}\right)^{2}} \frac{1}{\sqrt{\frac{\left(k+k^{\prime}\right)^{2}}{4}+4}} \ln \left\{\frac{1+\frac{i\left(k+k^{\prime}\right)}{4}+\frac{i}{2} \sqrt{\frac{\left(k+k^{\prime}\right)^{2}}{4}+4}}{1+\frac{i\left(k+k^{\prime}\right)}{4}-\frac{i}{2} \sqrt{\frac{\left(k+k^{\prime}\right)^{2}}{4}+4}}\right\}
\end{aligned}
$$

Let us divide the bulk contribution (4.26) in two parts: $B_{1}$ containing integrals over $k^{\prime \prime}$ of type (4.27) and $B_{2}$ whose $k^{\prime \prime}$ integrations are of type (4.28). For both it is necessary, after performing the integration over $\omega^{\prime \prime}$, to do the $k$ and $k^{\prime}$ integrals via closing contours in the upper half-plane to pick up the poles at $k$ or $k^{\prime}=\sqrt{\omega^{2}-4}=\hat{k}$. All other pole contributions lead to exponentially damped terms in the limit $x, x^{\prime} \rightarrow$ $-\infty$.

After some manipulation, $B_{1}$ is found to be equal to

$$
\begin{aligned}
B_{1}=-2 \beta^{2} \int & \frac{d \omega}{2 \pi} e^{-i \omega\left(t-t^{\prime}\right)} e^{-i \hat{k}\left(x+x^{\prime}\right)} \frac{1}{(2 \hat{k})^{2}} \frac{2 i \hat{k} \epsilon}{\left(i \hat{k}-2 \sigma_{1}\right)^{2}} \\
& \left\{-\frac{i}{4}+\frac{i a_{1}}{\sin a_{1} \pi} \frac{i \hat{k}}{i \hat{k}-2 \sigma_{1}}+\frac{1}{\pi} \frac{1}{\sqrt{\hat{k}^{2}+4}}\left(\frac{i \pi}{2}-\theta\right)\right\} .
\end{aligned}
$$

Notice that $B_{1}$, in the last term inside the braces, depends on $\theta$ in a manner which potentially is very inconvenient for a comparison with Ghoshal's formula. Fortunately, this term will be canceled by a matching term in $B_{2}$.

After somewhat lengthier calculations, $B_{2}$ is given by

$$
\begin{aligned}
B_{2}=-2 \beta^{2} \int \frac{d \omega}{2 \pi} e^{-i \omega\left(t-t^{\prime}\right)} e^{-i \hat{k}\left(x+x^{\prime}\right)} \frac{1}{(2 \hat{k})^{2}}\left\{\frac{-2 i \epsilon}{\left(i \hat{k}-2 \sigma_{1}\right)^{2}} \frac{\sigma_{1}^{2}}{\pi \sin ^{2} a_{1} \pi}\right. \\
+\frac{2 i \epsilon}{\left(i \hat{k}-2 \sigma_{1}\right)^{2}} \frac{a_{1} \sigma_{1}^{3}}{\sin ^{3} a_{1} \pi}+\frac{2 i \epsilon \hat{k}}{\left(i \hat{k}-2 \sigma_{1}\right)^{2}} \frac{1}{\pi \sqrt{\hat{k}^{2}+4}} \theta \\
\left.+\frac{i \hat{k}+2 \sigma_{1}}{i \hat{k}-2 \sigma_{1}}\left(-\frac{i \epsilon}{2 \pi \sin ^{2} a_{1} \pi}+\frac{i \epsilon a_{1} \sigma_{1}}{2 \sin ^{3} a_{1} \pi}\right)\right\} .
\end{aligned}
$$


Assembling the $O(\epsilon)$ part of (4.23) with (4.30) and (4.31) we obtain,

$$
\begin{aligned}
& -\frac{i \beta^{2} \epsilon}{2} \int \frac{d \omega}{2 \pi} e^{-i \omega\left(t-t^{\prime}\right)} e^{-i \hat{k}\left(x+x^{\prime}\right)}\left\{\frac{1}{\left(i \hat{k}-2 \sigma_{1}\right)^{2}}\left(\frac{1}{2 \pi}+\frac{a_{1} \sigma_{1}}{2 \sin a_{1} \pi}\right)\right. \\
& \left.+\frac{1}{\left(i \hat{k}-2 \sigma_{1}\right)^{3}}\left(-2 a_{1} \sin a_{1} \pi\right)+\frac{1}{\left(i \hat{k}-2 \sigma_{1}\right)^{2}} \frac{2}{\hat{k}}\left(-\frac{i}{4}+\frac{i}{2 \sqrt{\hat{k}^{2}+4}}\right)\right\},
\end{aligned}
$$

whence we can deduce the correction to the quantity $K_{1}$ in (4.13). Explicitly, we have,

$$
\begin{gathered}
\delta K_{1}=-i \beta^{2} \epsilon \hat{k}\left\{\frac{1}{\left(i \hat{k}-2 \sigma_{1}\right)^{2}}\left(\frac{1}{2 \pi}+\frac{a_{1} \sigma_{1}}{2 \sin a_{1} \pi}\right)+\frac{1}{\left(i \hat{k}-2 \sigma_{1}\right)^{3}}\left(-2 a_{1} \sin a_{1} \pi\right)\right. \\
\left.+\frac{1}{\left(i \hat{k}-2 \sigma_{1}\right)^{2}} \frac{2}{\hat{k}}\left(-\frac{i}{4}+\frac{i}{2 \sqrt{\hat{k}^{2}+4}}\right)\right\} .
\end{gathered}
$$

The correction to $K_{0}$ which was calculated before in [23] is,

$$
\begin{aligned}
\delta K_{0}=-\frac{i \beta^{2}}{8} K_{0}(\hat{k}) \sinh \theta\left\{\left(\frac{1}{\cosh \theta+1}-\frac{1}{\cosh \theta}\right)\right. \\
\left.+2 a_{1}\left(\frac{1}{\cosh \theta-\sin a_{1} \pi}-\frac{1}{\cosh \theta+\sin a_{1} \pi}\right)\right\} .
\end{aligned}
$$

This completes the collection of ingredients we need.

\section{Comparison with Ghoshal's formula}

In this section, the corrections to the classical reflection factor calculated above will be compared with the formula of Ghoshal quoted in (3.2).

Using (4.13), the relative correction to the classical reflection factor $K_{c}$ is given in terms of the corrections $\delta K_{0}$ and $\delta K_{1}$ by

$$
\frac{\delta K_{c}}{K_{c}}=K_{0}^{-1} \delta K_{0}+\epsilon\left(K_{0}^{-1} \delta K_{1}-K_{1} K_{0}^{-2} \delta K_{0}\right) .
$$

Hence, using (4.33) and (4.34) we have,

$$
\begin{aligned}
\frac{\delta K_{c}}{K_{c}}=-\frac{i \beta^{2}}{8} & \sinh \theta\left\{\left(\frac{1}{\cosh \theta+1}-\frac{1}{\cosh \theta}\right)\right. \\
+ & \left.2 a_{1}\left(\frac{1}{\cosh \theta-\sin a_{1} \pi}-\frac{1}{\cosh \theta+\sin a_{1} \pi}\right)\right\} \\
+ & \frac{i \beta^{2} \epsilon \sinh \theta}{8 \sin a_{1} \pi}\left\{\frac{1}{\pi}\left(\frac{1}{\cosh \theta-\sin a_{1} \pi}-\frac{1}{\cosh \theta+\sin a_{1} \pi}\right)\right. \\
+ & \left.a_{1} \cos a_{1} \pi\left(\frac{1}{\left(\cosh \theta-\sin a_{1} \pi\right)^{2}}+\frac{1}{\left(\cosh \theta+\sin a_{1} \pi\right)^{2}}\right)\right\} .
\end{aligned}
$$


On the other hand, Ghoshal's formula (3.2) for the reflection factor up to one loop order is given by:

$$
K_{q}(\theta) \sim K_{c}(\theta)\left(1-\frac{i \beta^{2}}{8} \sinh \theta \mathcal{F}(\theta)\right)
$$

where

$$
\begin{aligned}
\mathcal{F}(\theta)= & \frac{1}{\cosh \theta+1}-\frac{1}{\cosh \theta} \\
& +\frac{e_{1}}{\cosh \theta+\sin \left(e_{0} \pi / 2\right)}-\frac{e_{1}}{\cosh \theta-\sin \left(e_{0} \pi / 2\right)} \\
& +\frac{f_{1}}{\cosh \theta+\sin \left(f_{0} \pi / 2\right)}-\frac{f_{1}}{\cosh \theta-\sin \left(f_{0} \pi / 2\right)}
\end{aligned}
$$

In calculating (5.4) we have made use of the expansions of $E$ and $F$ to $O\left(\beta^{2}\right)$ :

$$
E \sim e_{0}+e_{1} \frac{\beta^{2}}{4 \pi} \quad F \sim f_{0}+f_{1} \frac{\beta^{2}}{4 \pi}
$$

with

$$
e_{0}=a_{0}+a_{1} \quad \text { and } \quad f_{0}=a_{0}-a_{1} .
$$

Since $K_{q}=K_{c}+\delta K_{c}$, we deduce that

$$
\frac{\delta K_{c}}{K_{c}}=-\frac{i \beta^{2}}{8} \sinh \theta \mathcal{F}(\theta)
$$

Thence, expanding to $O(\epsilon)$, we find,

$$
\begin{gathered}
\mathcal{F}(\theta)=\left\{\frac{1}{\cosh \theta+1}-\frac{1}{\cosh \theta}+\frac{e_{1}}{\cosh \theta+\sin a_{1} \pi}-\frac{e_{1}}{\cosh \theta-\sin a_{1} \pi}\right. \\
+\frac{e_{1} \epsilon \cos a_{1} \pi}{2 \sin a_{1} \pi}\left(\frac{1}{\left(\cosh \theta+\sin a_{1} \pi\right)^{2}}+\frac{1}{\left(\cosh \theta-\sin a_{1} \pi\right)^{2}}\right) \\
\left.+\frac{\epsilon f_{1}}{\sin a_{1} \pi \cosh ^{2} \theta}\right\} .
\end{gathered}
$$

Comparing (5.2) with (5.8) we see a pleasing similarity. In fact the two formulae are identical, to $O(\epsilon)$, provided we choose $e_{1}$ and $f_{1}$ suitably. In other words, we may deduce that

$$
e_{1}=-2 a_{1}+\frac{\epsilon}{\pi \sin a_{1} \pi} \equiv-\left(a_{0}+a_{1}\right)+O\left(\epsilon^{2}\right),
$$

and $f_{1}$ is proportional to $\epsilon$. Unfortunately, the calculation does not allow anything more detailed to be learned about $f_{1}$. To do better would need a correction to the reflection factor to $O\left(\epsilon^{2}\right)$. 


\section{Discussion}

The purpose of this calculation was to test a little more deeply the expression for the sinh-Gordon particle reflection factor given in [2] and to learn additional information concerning its dependence on the boundary parameters $\sigma_{0}$ and $\sigma_{1}$. The result of the investigation is gratifying because it agrees with alternative derivations of the reflection factor and it also agrees with the following conjecture. Everything we have learned so far is consistent with quite simple expressions for $E$ and $F$ :

$$
E=\left(a_{0}+a_{1}\right)(1-B / 2) \quad F=\left(a_{0}-a_{1}\right)(1-B / 2),
$$

where the coupling constant dependence enters via the expression for $B$ given in (2.4). Similar expressions for these parameters have been arrived at via other arguments by Zamolodchikov 33].

If (6.1) is correct then the reflection factor is invariant under the interchange $a_{0} \leftrightarrow$ $a_{1}$. In effect, this invariance restores the $\mathbf{Z}_{\mathbf{2}}$ bulk symmetry which apparently was broken by the boundary condition and replaced by a symmetry under the simultaneous interchange of $\phi$ with $-\phi$ and $a_{0}$ with $a_{1}$. The reflection factor is also invariant if $a_{0}$ and/or $a_{1}$ is replaced by its negative, as it should be given the definitions of $\sigma_{0}$ and $\sigma_{1}$. It is consistent with what is known at the special value of the coupling constant, known as the 'free-fermion' point in the sine-Gordon model, where $B=-2$ and the S-matrix is unity. There, the restrictions on the parameters in the reflection factor can be solved exactly and are in agreement with (6.1) [23].

Note also, that with the expressions (6.1) the reflection factor (3.2) has a weakstrong coupling symmetry matching the symmetry of the S-matrix under $\beta \rightarrow 4 \pi / \beta$. To see this, note that setting

$$
\left(a_{0}^{*}, a_{1}^{*}, \beta^{*}\right)=\frac{4 \pi}{\beta^{2}}\left(a_{0}, a_{1}, \beta\right)
$$

defines a new triple of coupling constants with the property that

$$
K_{q}\left(\theta, a_{0}, a_{1}, \beta\right)=K_{q}\left(\theta, a_{0}^{*}, a_{1}^{*}, \beta^{*}\right) .
$$

If (6.1) is correct, implying the duality symmetry (6.2), then we are faced with other puzzles. For example, it is known that the supersymmetric version of the sinh-Gordon model is only integrable when restricted to a half-line with some very special boundary conditions (either $a_{0}=a_{1}=0$ or, $a_{0}=a_{1}=1$ ) (see [34]), and this restriction would appear to be incompatible with a weak-strong coupling symmetry without modifying (6.1).

It is also known [6, 8] that the other affine Toda field theories constructed from data in the ade series, when restricted to a half-line, allow only a finite number of possible boundary conditions. In fact, the $a_{1}^{(1)}$ or sinh-Gordon model is apparently the only example within this series which allows continuous boundary parameters. Expressions for the associated reflection factors for the other models are largely unknown but, it will be interesting to discover if they too can permit a duality symmetry in the presence of a boundary which will match the symmetry of their bulk S-matrices. 


\section{Acknowledgement}

One of us (AC) wishes to thank the Ministry of Culture and Higher Education of Iran for financial support. The other (EC) thanks G.W. Delius and Al.B. Zamolodchikov for discussions and the European Commission for partial support under a TMR grant, number FMRX-CT96-0012.

\section{References}

[1] S. Ghoshal and A. Zamolodchikov, 'Boundary S matrix and boundary state in two-dimensional integrable quantum field theory, Int.J.Mod.Phys. A9 (1994) 3841.

[2] S. Ghoshal, 'Boundary state boundary S-matrix of the sine-Gordon model', Int.J.Mod.Phys. A9 (1994) 4801.

[3] A. Fring and R. Koberle, 'Factorized scattering in the presence of reflecting boundaries', Nucl.Phys. B421 (1994) 159.

[4] A. Fring and R. Koberle, 'Affine Toda field theory in the presence of reflecting boundaries', Nucl.Phys. B419 (1994) 647.

[5] R. Sasaki, 'Reflection bootstrap equations for Toda field theory', in Interface between physics and Mathematics, eds. W. Nahm and J-M Shen, (World Scientific 1994) 201.

[6] E. Corrigan, P.E. Dorey, R.H. Rietdijk and R. Sasaki, ' Affine Toda field theory on a half-line' Phys.Lett. B333 (1994) 83.

[7] E. Corrigan, P.E. Dorey, R.H. Rietdijk, 'Aspects of affine Toda field theory on a half-line', Prog.Theor.Phys.Suppl. 118 (1995) 143; hep-th/9407148.

[8] P. Bowcock, E. Corrigan, P.E. Dorey and R.H. Rietdijk, 'Classically integrable boundary conditions for affine Toda field theory', Nucl.Phys B445 (1995) 469.

[9] S. Skorik and H. Saleur, 'Boundary bound states and boundary bootstrap in the sine-Gordon model with Dirichlet boundary condition', J.Phys. A28 (1995) 6605.

[10] A. Fujii and R. Sasaki, 'Boundary effects in integrable field theory on a half-line', Prog.Theor.Phys. 93 (1995) 1123; hep-th/9503083.

[11] P. Bowcock, E. Corrigan, R.H. Rietdijk, 'Background field boundary conditions for affine Toda field theories', Nucl.Phys. B465 (1996) 350; hep-th/9510071.

[12] S. Penati, D. Zanon, 'Quantum integrability in two-dimensional system with boundary', Phys.Lett. B358 (1995) 63.

[13] P. Bowcock, 'Classical backgrounds and scattering for affine Toda theory on a half-line', JHEP 05 (1998) 008; hep-th/9609232.

[14] E. Corrigan, 'Integrable field theory with boundary conditions', Frontiers in Quantum Field Theory, eds Chao-Zheng Zha and Ke Wu, (World Scientific 1998) 9; hepth/9612138. 
[15] G.W. Delius, Restricting affine Toda field theory to the half-line', JHEP 9809 (1998) 016; hep-th/9807189.

[16] G.M.Gandenberger, 'On $a_{2}^{(1)}$ reflection matrices and affine toda theories', Nucl. Phys. B542 (1999) 659; hep-th/9806003

[17] G.W. Delius and G.M. Gandenberger, 'Particle reflection amplitudes in $a_{n}^{(1)}$ Toda field theories', Nucl. Phys. B554 (1999) 325; hep-th/9904002

[18] M. Perkins and P. Bowcock, 'Quantum corrections to the classical reflection factor in $a_{2}^{(1)}$ Toda field theory', Nucl.Phys. B538 (1999) 612.

[19] E. Corrigan and G.W. Delius, 'Boundary breathers in the sinh-Gordon model', J.Phys. A32 (1999) 8601; hep-th/9909145

[20] R. Rajaraman, Solitons and Instantons (North Holland 1982).

[21] J.D. Kim, 'Boundary reflection matrix in perturbative quantum field theory', Phys.Lett. B353 (1995) 213.

[22] J.D. Kim, 'Boundary reflection matrix for A-D-E affine Toda field theory', hepth/9506031.

[23] E. Corrigan, 'On duality and reflection factors for the sinh-Gordon model with a boundary', Int.J.Mod.Phys. A13 (1998) 2709; hep-th/9707235.

[24] N. Topor, 'Perturbation method for boundary S-matrix in 2D quantum field theory', Mod.Phys.Lett. A12 (1997) 2951.

[25] L.D. Faddeev and V.E. Korepin, 'Quantum theory of solitons', Phys. Rep. 42 (1978) 1.

[26] A.B. Zamolodchikov and Al.B. Zamolodchikov, 'Factorised S-matrices in two dimensions as the exact solutions of certain relativistic quantum field theory models', Ann. Phys. 120 (1979) 253.

[27] A.E. Arinshtein, V.A. Fateev and A.B. Zamolodchikov, 'Quantum S-matrix of the 1+1 dimensional Toda chain', Phys.Lett. B87 (1979) 389.

[28] K. Symanzik, 'Schrodinger representation and casimir effect in renormalization quantum field theory', Nucl.Phys. B190 (1981) 1.

[29] H.W. Diehl and S. Dietrich, 'Multicritical behaviour at surfaces' Z.Phys. B50 (1983) 117.

[30] M. Benhamou and G. Mahoux, 'Fluctuations and renormalization of a field on a boundary', Nucl.Phys. B305 (1988) 1.

[31] H.W. Braden, E. Corrigan, P.E. Dorey and R Sasaki, 'Affine Toda field theory and exact S-matrices', Nucl. Phys. B338 (1990) 689.

[32] E. Corrigan, 'Recent developments in affine Toda quantum field theory', CRMCAP Summer School on Particles and Fields '94, Banff, eds. G. Semenoff, L. Vinet (Springer New York) 1999; hep-th/9412213 
[33] Al.B. Zamolodchikov, unpublished private communication.

[34] T. Inami, S. Odake and Y. Zhang, 'Supersymmetric extension of the Sine-Gordon theory with integrable boundary interactions,' Phys. Lett. B359 (1995) 118; hepth/9506157. 